\title{
Evaluation of Generalized Linear Mixed Models for Analyzing Disease Incidence Data Obtained in Designed Experiments
}

\author{
L. V. Madden, Department of Plant Pathology, Ohio State University, Wooster 44691; W. W. Turechek, Department of \\ Plant Pathology, New York State Agricultural Experiment Station, Cornell University, Geneva, NY 14456; and M. Nita, \\ Department of Plant Pathology, Ohio State University, Wooster 44691
}

\begin{abstract}
Madden, L. V., Turechek, W. W., and Nita, M. 2002. Evaluation of generalized linear mixed models for analyzing disease incidence data obtained in designed experiments. Plant Dis. $86: 316-325$

Diseased individuals (e.g., leaves, plants) typically are clustered in nature, resulting in greater heterogeneity or variability of disease incidence than would be expected for a random pattern. To account for this variability, as well as the binary nature of disease incidence and the multiple sources of variation in designed experiments, a generalized linear mixed model (GLMM) can be used to analyze collected data. GLMMs are becoming more common in many disciplines and may be preferred over analysis of variance for non-normally distributed data. We evaluated several GLMMs for analyzing the incidence of Phomopsis leaf blight of strawberry in relation to fungicide treatments in five experiments which varied greatly in mean incidence and the differences in incidence between treatments. The first model form accounted for heterogeneity through the residual variance (i.e., the overdispersion parameter), which was assumed to be either fixed for the experiment, or dependent on either treatment or incidence. The second model form accounted for heterogeneity explicitly through a within-plot sampling variance, which was assumed to be either constant or dependent on treatment. All GLMMs could be successfully fitted to the data in each experiment, but there was weak evidence based on the conditional deviance and residual plots that the residual-variance models were more appropriate than the sampling-variance models. Model choice had only a minor effect on $F$ tests for treatment effects and significant differences between treatment means. Based on ease of use and evaluation results, we recommend that the simplest (fixed residual variance) model be used as the first choice in analyzing disease incidence data using GLMMs.
\end{abstract}

Proper statistical analysis of designed experiments is essential in order to appropriately evaluate control strategies and tactics. For many decades, analysis of variance (ANOVA) has been the traditional method for determining the effects of experimental factors, such as fungicide treatments, cultivars, and other control procedures, on disease intensity $(9,30)$. In recent years, however, there have been many statistical advances made on using mixed linear models for analyzing such experiments $(11,40,41)$. We refer to this analysis as a mixed model ANOVA. This approach formally handles the analysis of experiments with both fixed (e.g., fungi-

Corresponding author: L. V. Madden

E-mail: madden.1@osu.edu

* The $\boldsymbol{e}$-Xtra logo stands for "electronic extra" and indicates the HTML abstract available on-line contains supplemental material not included in the print edition.

Accepted for publication 5 November 2001.

Publication no. D-2002-0111-03S

(C) 2002 The American Phytopathological Society cide treatment) and random (e.g., block, location) effects, in that tests for factor and interaction effects are properly performed, and the standard errors of means and mean differences are calculated correctly. The "standard" ANOVA conducted by most statistical programs is really a fixedeffects-only method, even when random effects are specified, with some postmodel-fitting adjustments to calculate expected mean squares for the factors and interactions that involve random effects (23). Compared with standard ANOVA, mixed model ANOVA is computationally intensive, so it is not surprising that the latter approach has increased in popularity as high-speed personal computers have become common and efficient algorithms have been written.

Plant diseases often are measured in the form of incidence, the proportion or percentage of plants (or plant units) diseased relative to the number of plants (or plant units) observed (26). Incidence is a binary variable because each observed individual is either diseased or not. Incidence is characterized by a binomial or beta-binomial distribution, not a normal distribution (an assumption of ANOVA), and the variance of incidence is a function of the mean $(12,13)$. The variance typically is higher than expected for a binomial distribution and often can be represented by a power function of disease incidence, which is known as the binary power law (26). Although it is acceptable to use fixed-effects ANOVA to analyze incidence if a proper transformation of the proportion of diseased individuals is made and there is a large number of observations for each sampling unit, many statisticians argue that one should use a generalized linear model for the analysis $(5,14,28,29)$. Tests of significance, standard errors, and contrasts of the means can be affected if ANOVA is used instead of a generalized linear model appropriate for binary data. Furthermore, so-called variance-stabilizing transformations (such as the angular transform of proportions) may not, in fact, fully stabilize variances across the full range of incidences when some of the means are close to 0 and $100 \%$. It should be emphasized that a generalized linear model is much broader in scope than a standard ANOVA or linear regression model; in fact, fixedeffects ANOVA and regression are special cases of generalized linear models. Collett (5) provides a thorough discussion of generalized linear models for binary data, and Hughes and Madden (14) discuss at length the analysis of disease incidence with generalized linear models.

Use of standard generalized linear models is most suitable for completely randomized experimental designs where there is only one error (residual) variance. For randomized complete blocks, split-plots, and other complicated designs that involve random and fixed factors and two or more sources of variation, generalized linear models are not fully satisfactory. Just as standard ANOVA has been expanded to mixed model ANOVA, recent research has expanded generalized linear model analysis to generalized linear mixed model (GLMM) analysis (2,42). This statistical approach is now becoming common in many disparate fields $(6,7,10,24)$. Although computationally intensive, GLMMs can, in principle, deal with the statistical properties of disease incidence and account for multiple random and fixed factors in the analysis. 
When using mixed model ANOVA to analyze plant disease incidence data, one essentially is manipulating the data to make them fit a model that is less than ideal. As stated by Kachman (17), "it seems more reasonable to start with an appropriate model for the data and use an estimation procedure derived from that model." Now that efficient computer algorithms are available, it is possible to fit a GLMM to data sets containing random effects, nonnormal error distributions, and higher variability than expected for a binomial distribution (42). Furthermore, one can directly account for certain aspects of the statistical distribution of diseased individuals, such as the relationship between the mean and variance (12), the discrete nature of the analyzed variable (26), and the multiple sources of variation in many planned experiments $(2,33)$. However, the parameter estimation method involves some approximations and depends on assumptions about the distribution of the random effects and a still-evolving statistical theory, so it is not clear in general which specific models should be used under various circumstances and whether or not specific models will adequately describe the data $(23,29)$. For instance, recent research indicates that the GLMM can give biased results when the number of observations per sampling unit $(n)$ is very small, such as 4 or less (21).

Piepho (33) recently showed how to analyze disease incidence data from a randomized complete block design using GLMMs. Two different approaches (model forms) were considered to account for the variability within the experimental units (i.e., plots), one directly through the determination of the sampling variance, and the other indirectly through the determination of the residual variance (i.e., variability not explained by any of the explicit fixed- and random-effects terms in the model). In standard form, both approaches assumed a fixed value for the variability for all levels of the fixed factors. Because it is well established that the variability or heterogeneity of incidence in fields or plots is related to the mean incidence $(15,26,39)$, Piepho (33) followed the lead of Hughes and Madden (14) and also analyzed the incidence data using both GLMM approaches assuming that the within-plot variability or residual variation depended on the treatment level. However, no general conclusions have yet been drawn regarding the superiority of any of these models for analyzing disease incidence data.

The objective of this study was to evaluate various GLMMs for analyzing disease incidence data collected in designed experiments with more than one source of variation. To accomplish this objective, experiments over 5 years were analyzed for the effects of fungicides on the control of Phomopsis leaf blight of strawberry, caused by Phomopsis obscurans. The model forms of Piepho (33) were considered, with and without treatmentdependent variability. We also show here for the first time how to directly incorporate into the GLMM-type analysis the previously determined binary power law relationship between the variance and the mean (12). To do this, we use the methodology developed for heterogenous variances in general linear and mixed models (4).

\section{MATERIALS AND METHODS}

Data sets. Experiments were conducted at a commercial farm near Wooster, $\mathrm{OH}$, from 1996 to 2000. Strawberry plants were grown in a matter-row system, with $\sim 1 \mathrm{~m}$ between row centers, and were at least 3 years old. In each year, a fairly uniform section of a field of strawberries was selected, consisting of 8 to 12 rows, depending on the number of treatments. The field section was divided into four blocks down each row, so that all 8 to 12 rows were in each block. Row length in each block was $3 \mathrm{~m}$, and there was a 1-m buffer of plants between blocks. Fungicide treatments were randomly assigned to the rows in each block separately.

Numbers of fungicide treatments $(t)$, including an unsprayed control, were 12 (1996), 8 (1997), 9 (1998), and 10 (1999 and 2000). Except for 1996, the experiments were done in the spring and early summer, which is the time of flowering, fruit production, and harvest for the Junebearer cultivars grown in Ohio. Commercial disease management focuses on fungicide use from flowering to harvest (8). The 1996 experiment was done in mid-to-late summer, in order to evaluate fungicide effects on disease development during the regrowth phase of strawberries after postharvest renovation in which plants are mowed to soil level.

The fungicides and rates of application tested varied from year to year. Summaries of the individual experiments have been published, except for $1996(31,32,37,38)$. Fungicides were applied until runoff using a $\mathrm{CO}_{2}$-pressurized hand-held sprayer operated at a pressure of $276 \mathrm{kPa}$. Fungicide applications were made on a 7- to 14-day schedule, depending on the year, which resulted in four to six spray applications in a given year.

Disease assessment was done by choosing either three $(1999,2000)$ or five (1996 to 1998) equally spaced sampling units per plot (each treatment of each block). Sampling units consisted of 15 strawberry leaflets. Each leaflet was observed for symptoms of leaf blight, and the disease status (i.e., diseased or not) of each leaflet was recorded. Thus, there could be from 0 to 15 diseased leaflets per sampling unit. Disease assessments were typically done at least twice, but only data from one time were analyzed here. In 1999 and 2000, sampling units also were vertically divided into lower and upper halves (upper and lower parts of the canopy), with 15 leaflets assessed in each half. Only the data from the lower half of each sampling unit were analyzed here to be consistent with other years. Results previously reported sometimes corresponded to a different assessment time than the one used here, or were based on pooling the data from the upper and lower halves of the canopies.

Generalized linear mixed models. The experimental design for each experiment was a randomized complete block, with treatment $(\tau)$ as a fixed effect, and block $(B)$ and experimental (plot) error as random effects. We use the $i$ subscript to represent the specific treatment (e.g., $i=1, \ldots$, $t)$ and $j$ for the specific block $(j=1, \ldots, 4)$. We use $p$ to represent the probability of a leaflet being diseased, $X$ the number of diseased leaflets, $n$ the number of leaflets in the sampling unit, and $x$ the proportion diseased $(X / n)$. For a single sample of $n=$ 15 leaflets and $X=5$ diseased leaflets, an estimate of $p$ is $\hat{p}=x=5 / 15=0.33$. In generalized linear models and GLMMs for binary data (such as disease incidence), it is usually first assumed that $X$ has a binomial distribution with parameters $p$ and $n$. The variance of $x, \operatorname{Var}(x)$, is given by $p(1-$ $p) / n$. If there is greater variability than predicted by the binomial distribution (i.e., overdispersion or extra-binomial variation), it is possible to simply assume that disease incidence has an unspecified distribution with mean (expected value) of $p$ and a variance proportional to $p(1-p) / n$. In this case, so-called quasi-likelihoods are calculated instead of likelihoods (5).

In generalized linear models and GLMMs, a function of the expected value of $x$ ( $p$ in this case) is modeled in relation to the experimental factors. This function of $p$ is known as the link; for binary data, the logit link is typically used. The logit of $p$, logit $(p)$, is given by $\ln [p /(1-p)]$.

A GLMM for the strawberry fungicide experiments is:

$\operatorname{logit}\left(p_{i j}\right)=\eta_{i j}=\mu+\tau_{i}+B_{j}+(\tau B)_{i j}$

in which $p_{i j}$ is the probability of disease for the $i$ th treatment in the $j$ th block, $\mu$ is a constant, $\tau_{i}$ is the effect of the $i$ th treatment, $B_{j}$ is the effect of the $j$ th block, and $(\tau B)_{i j}$ is the experimental error of the $i j$ th plot (notationally written as the treatment by block interaction). It is assumed that the block effect and the experimental error are normally distributed. We call this Model A, which corresponds to Model 3 of Piepho (33). The term $\eta_{i j}$ is known as the linear predictor in the terminology of generalized linear models. When the parameters or terms $\left.\left[\tau_{i}, B_{j},(\tau B)_{i j}\right)\right]$ are estimated, one obtains the estimated linear predictor, $\hat{\eta}_{i j}$; then the estimated probability of disease conditional on the random effects is given by

$$
\hat{p}_{i j}=1 /\left[1+\exp \left(-\hat{\eta}_{i j}\right)\right]
$$


The transformation of $\hat{\eta}_{i j}$ is known as the inverse link and is used to estimate the probability of a leaflet being diseased. This estimate of $p$ is not necessarily the same as simple proportion of diseased leaflets in the $i j$ th plot.

Model fitting (see below) results in estimates of the fixed- and random-effect terms, as well as the variance for blocks $\left(\sigma_{B}^{2}\right)$ and the experimental error $\left(\sigma_{\tau B}^{2}\right)$. The variability of disease incidence among sampling units within plots (the sampling error) is not explicitly considered as a term in equation 1. Because this variability is not accounted for, the variance of incidence is expected to be large (inflated) relative to the binomial variance $\left[p_{i j}(1-\right.$ $\left.\left.p_{i j}\right) / n\right]$. Specifically, the inflation of the variance of $x_{i j}$ (conditional on the random effects), is then given by $\operatorname{Var}\left(x_{i j}\right)=\phi p_{i j}(1-$ $\left.p_{i j}\right) / n$, in which $\phi$ is typically known as the overdispersion parameter. This parameter is also estimated by the model fitting procedure as the residual variance. When $\phi=$ 1 , there is no variance inflation, an indication that the probability of disease is constant among sampling units within plots.

An alternative to Model A is to explicitly account for variation in disease among sampling units within plots. A GLMM for the experiments is:

$\operatorname{logit}\left(p_{i j k}\right)=\eta_{i j k}=\mu+\tau_{i}+B_{j}+(\tau B)_{i j}+e_{i j k}$

in which $p_{i j k}$ is the probability of disease for the $i$ th treatment, $j$ th block, and $k$ th sampling unit $[k=1, \ldots, 3$ (or 5 , depending on the year)], and $e_{i j k}$ is the effect of the $k$ th sampling unit in the $i j$ th plot (sampling error). Blocks, experimental error, and sampling error are all considered to be random effects which follow a normal distribution. We call this Model B, which corresponds to Model 2 of Piepho (33). The model fitting results in estimates of the fixed- $\left(\tau_{i}\right)$ and random-effect terms $\left[B_{j}\right.$, $\left.(\tau B)_{i j}, e_{i j k}\right]$, as well as the variance for blocks $\left(\sigma_{B}^{2}\right)$, experimental error $\left(\sigma_{\tau B}^{2}\right)$, and the within-plot sampling error $\left(\sigma_{e}^{2}\right)$. For this model, it is assumed that the explicit terms in the model account for all variability in disease incidence, so that the residual variance equals the binomial variance, and, hence, $\phi=1$.

Heterogeneous variances. Both Models $\mathrm{A}$ and $\mathrm{B}$ can be expanded to account for variability that depends on treatment. Such dependency may be needed since a considerable body of literature indicates that the variation of disease incidence within fields or plots is a nonlinear function of the expected disease incidence $(12,15,26)$. If treatment affects the mean, then presumably treatment will also affect the variability (either $\phi$ or $\sigma_{e}^{2}$ ). Model A (eq. 1) is expanded by specifying a treatment-specific overdispersion parameter (residual variance) for each treatment level, $\phi_{i}$. We call this model C. Model B is expanded by specifying a treatment-specific (withinplot) sampling variance, $\sigma_{e, i}^{2}$. We call this
Model E. In both cases, the number of variance terms that have to be estimated increases by $t-1$.

Although Models C and E provide general ways of dealing with heterogenous variances, a large number of parameters may need to be estimated if there are many treatments, possibly resulting in difficulties in model fitting and interpretation. An alternative approach is to expand Model A by utilizing the binary power-law relationship for the heterogeneity of disease incidence (15). Suppressing subscripts for convenience, the variance of disease incidence can be written as:

$\operatorname{Var}(x)=A[p(1-p) / n]^{b}$

in which $A$ and $b$ are parameters. If the binomial distribution describes the data, $b$ $=1$ and $A=1$. Recalling that $\operatorname{Var}(x)$ can also be expressed as $\phi p(1-p) / n$, one can write equation 3 as:

$$
\operatorname{Var}(x)=A[p(1-p) / n]^{b-1}[p(1-p) / n]
$$

which indicates that one can define $\phi$ as a function of $p, \phi(p)$, and write it as:

$\phi(p)=A[p(1-p) / n]^{b-1}$

Using this formulation, one can model $\phi$ as a function of expected probabilities for individual treatments, $\phi\left(p_{i}\right)$, or expected probabilities for individual plots, $\phi\left(p_{i j}\right)$. We consider only the former here. For simplicity, we write $b^{\prime}$ for $b-1$. When $b^{\prime}$ $=0$, equation 4 reduces to a constant $A$. We call equation 1 with $\phi$ defined by equation 4 as Model D. In comparison with Model A, one parameter is added in Model D to represent nonconstant residual variability (i.e., the single $\phi$ is replaced by $A$ and $b^{\prime}$ ).

We thus compared five GLMMs for analyzing the strawberry leaf blight data over 5 years. All models specify a random effect for block and experimental error. Models A, C, and D all follow from equation 1, with three ways of specifying residual variability. Models B and E follow from equation 2, with two ways of specifying $\sigma_{e}^{2}$. Models that assume no overdispersion and no within-plot variability (i.e., $\phi=1$ and $\sigma_{e}^{2}=0$ ) are not considered here, because it is well established that this assumption can lead to large errors in statistical tests and calculated standard errors of estimated parameters (14).

Model fitting. Models were fitted to the data using restricted pseudo-likelihood (REPL), as developed by Wolfinger and O'Connell (42) and described in detail in Littell et al. (23). The \%GLIMMIX macro of the Statistical Analysis System (SAS version 8; SAS Institute, Cary, NC) was used to perform the REPL procedure. Piepho (33) discusses the use of the macro in some detail. Essentially, \%GLIMMIX is based on iterative use of the MIXED procedure of SAS, which, among other things, fits mixed linear models using restricted maximum likelihood.
For \%GLIMMIX (as with MIXED), one designates the fixed effects, the random effects, and when there is a random effect for within-plot variability (Models B and E), indicates whether a fixed samplingvariance term (Model B) or treatmentdependent sampling-variance term (Model $\mathrm{E})$ is to be used. It is assumed that there is a single unknown residual variance term ( $\phi)$ to be estimated, but this term is restricted to equal 1 when Models B and E are being fitted (otherwise the model would be over-parameterized). Moreover, $\phi$ can be specified as treatment-dependent $\left(\phi_{i}\right.$; Model C) or a function of terms such as disease incidence (Model D). For the latter, the convention of \%GLIMMIX (and MIXED) is to specify $\phi$ (with the LOCAL option of the REPEATED statement) as an exponential function of one or more variables, following the methods given in Carroll and Ruppert (4). For example, with an arbitrary variable, $z$, one can write $\phi$ as $A \cdot \exp (\varphi z)$, in which $A$ and $\varphi$ are parameters. This formulation assures that predicted variances are nonnegative for any $z$. In order to write equation 4 in this form, one defines $z=\ln [p(1-p) / n]$. Then, $\varphi=b$ $-1=b^{\prime}$ of equation 4 . The estimates of $p$ are used in $z$, but these estimates are not known until the model fitting is complete. Thus, a two-step process is invoked. First, a version of Model A is fitted with treatment as the fixed and only factor in the model. The estimated $p$ 's for the treatments $\left(\hat{p}_{i}\right)$ are stored together with the original data. Second, Model D is fitted, using the original data, and with $z$ calculated using the estimated $p$ 's.

$F$ tests based on scaled Wald statistics were used for determining if treatment significantly affected the probability of a leaflet being diseased (23). The significance level of the $F$ tests $(P)$, the estimated linear predictors for each treatment evaluated at the expected values of the random effects $\left[\hat{\eta}_{i}\right.$; i.e., $\left.\operatorname{logit}\left(\hat{p}_{i}\right)\right]$, and the standard errors (SEs) of the estimated linear predictors were compared among models to determine the effects of the model choice on analysis of the treatment effects. In the convention of the SAS procedure, the estimated linear predictors are known as the least squares means. (Note that the estimate of $p$ for treatment $i$ at the expected value of block and experimental error [eq. 1] more completely is written as $\hat{p}_{i \bullet}$, and the estimate at the expected value of block, experimental error, and within-plot sampling error [eq. 2] is written as $\hat{p}_{i \bullet \bullet}$; however, we suppress the "dot" subscripts for $j$ and $k$ positions for convenience of expression.) The standard errors of the difference (SED) were also calculated for all pairs of treatments and used to determine significant differences in estimated linear predictors between treatments (23).

Model evaluation. For GLMMs, there is no single test to indicate which model is most appropriate. This is partly because the 
parameter estimation method depends on several approximations and so-called conditional expectations (23), and it is not always known how accurate the approximations are for small to medium size data sets. Moreover, the statistical theory for certain aspects of GLMMs has not been fully resolved. For generalized linear models, the deviance $(D)$ is of central importance for determining goodness of fit and for testing (fixed) effects $(5,22) . D$ equals -2 times the (likelihood [or quasilikelihood] for the fitted model minus the likelihood [or quasi-likelihood] for the saturated model [in which the fitted values equal the observations]). If there is a good fit with generalized linear models, the expected value of $D$ equals the degrees of freedom $(v)$, that is, the number of observations in the data set minus the number of (fixed-effect) parameters estimated. For GLMMs, however, the quasi-likelihood calculated for the REPL method is conditional on the random effects in the model (with different random terms or parameter estimates for each model). Thus, comparing $D$ to $v$ does not provide an absolute evaluation of the model fit, but does provide a convenient means of comparing the fit of different GLMMs conditional on the random effects (23). We calculated the ratio $D / v$ for each model and year. Note that the number of random-effects parameters does not enter the calculation of $v$.

Models were also compared based on residual plots. Omitting subscripts for convenience, standardized Pearson residuals were calculated for each observation as:

$$
r=\frac{(x-\hat{p}) \sqrt{n}}{\sqrt{\hat{\phi} \hat{p}(1-\hat{p})(1-h)}}
$$

in which $h$ is the so-called "leverage" for the observation and a function of the variance of the estimated linear predictor $(\hat{\eta})$ (see page 329 in citation 5 for details on calculating the leverage). For equation 2 (Models B and E), $\phi$ equals 1 by definition. For each model and year, $r$ was plotted versus the estimated linear predictor $(\hat{\eta})$ conditional on the random effects. A good fit is indicated by a random scatter of points of the residuals.

\section{RESULTS}

Disease incidence data. Mean disease incidence varied considerably over the 5 years (Table 1). Mean incidence in the unsprayed plots ranged from a low of 0.29 (1996) to a high of 0.99 (1999). In 1996, means did not vary much among treatments, but in 1997 and 2000 there were large differences among the treatments. Therefore, the experiments over the 5 years encompassed a wide range of incidences of disease, indicating that statistical results should be reflective of many typical field studies that evaluate the effects of control measures on disease incidence.
Model fits. The REPL method was able to converge for all five models over 5 years, and test statistics and standard errors could be determined in all cases. The conditional deviance $(D)$ values in fitting Models A, C, and D to the data were considerably larger than $v$, indicating overdispersion that could be attributable to sampling variation within plots (Table 2). Values of $D / v$ for 1996 to 2000 corresponding to Model A were between 1.4 and 2.3. Similar values were found for Models C and D. For Model A, overdispersion was accounted for by the estimate of $\phi$, which ranged from 1.4 to 2.2 (Table 3 ). It should be noted that with generalized linear models, one way of calculating $\phi$ is by $D / v$ (5). However, the REPL procedure for GLMMs directly estimates $\phi$, and this estimate is not necessarily in agreement with $D / v$, especially for small to medium sized data sets. The closeness found here between $\hat{\phi}$ and $D / \nu$ is an indication that Model $\mathrm{A}$ is providing at least a reasonable way of accounting for the overdispersion, although the closeness does not mean that Model A is providing a good fit in general.

The deviances for Models B and E, in which an explicit term for within-plot variability (eq. 2) was specified, were lower than for Models A, C, and D. Values of $D / v$ corresponding to Model B for 1996 to 2000 were between 0.6 and 0.7 (Table 2). These values were all considerably less than the assumed $\phi$ of 1 , an indication of underdispersion (less residual variability than expected) when the additional random-effects term was included. Estimates of $\sigma_{e}^{2}$ ranged from 0.28 to 0.77 over the 5 years (Table 3 ). When Model D was fitted to the data, estimates of $b^{\prime}$ for the binary power law function (eq. 4) ranged from 0.28 to 0.75 over the 5 years (Table 3 ). These estimates were more than twice as large as their (large-sample-theory-based) standard errors in 1997, 1998, and 1999. Estimates of $A$ (eq. 4) also varied greatly over the 5 years. Although $A$ is interpreted as the single $\phi$ (Model A) when $b^{\prime}=0, A$

Table 1. Mean disease incidence ${ }^{\mathrm{a}}$ of strawberry leaf blight in commercial strawberry plots over 5 years for different fungicide treatments

\begin{tabular}{lccccc}
\hline & \multicolumn{5}{c}{ Year } \\
\cline { 2 - 6 } Treatment $^{\mathbf{b}}$ & $\mathbf{1 9 9 6}^{\mathbf{c}}$ & $\mathbf{1 9 9 7}$ & $\mathbf{1 9 9 8}$ & $\mathbf{1 9 9 9}$ & $\mathbf{2 0 0 0}$ \\
\hline 1 & 0.21 & 0.30 & 0.11 & 0.98 & 0.26 \\
2 & 0.14 & 0.28 & 0.10 & 0.88 & 0.43 \\
3 & 0.15 & 0.15 & 0.14 & 0.94 & 0.25 \\
4 & 0.29 & 0.15 & 0.10 & 0.88 & 0.32 \\
5 & 0.23 & 0.03 & 0.07 & 0.64 & 0.41 \\
6 & 0.22 & 0.08 & 0.08 & 0.89 & 0.26 \\
7 & 0.32 & 0.03 & 0.003 & 0.90 & 0.28 \\
8 & 0.41 & 0.46 & 0.10 & 0.90 & 0.16 \\
9 & 0.31 & & 0.28 & 0.91 & 0.01 \\
10 & 0.30 & & & 0.99 & 0.51 \\
11 & 0.30 & & & & \\
12 & 0.29 & & & & \\
Mean & 0.26 & 0.19 & 0.11 & 0.89 & 0.29 \\
\hline
\end{tabular}

a Proportion of leaflets with symptoms of leaf blight caused by Phomopsis obscurans. Each treatment mean is based on 4 blocks of 5 (1996-1998) or 3 (1999-2000) sampling units each, with each sampling unit consisting of 15 leaflets.

${ }^{\mathrm{b}}$ Treatment label corresponds to fungicide application regime. The last treatment within a year is the unsprayed control. Treatment labels are not the same from year to year.

${ }^{c}$ The experiment in 1996 was different from the rest in that the fungicides were applied only in mid to late summer (after harvest and renovation); in the other years, the fungicides were applied in the spring before harvest.

Table 2. Conditional deviance divided by the degrees of freedom $(D / v)^{\mathrm{a}}$ for the fit of generalized linear mixed models (GLMMs) to the strawberry leaf blight data over 5 years

\begin{tabular}{lccccc}
\hline & \multicolumn{5}{c}{ Year } \\
\cline { 2 - 6 } Model $^{\mathbf{b}}$ & $\mathbf{1 9 9 6}$ & $\mathbf{1 9 9 7}$ & $\mathbf{1 9 9 8}$ & $\mathbf{1 9 9 9}$ & $\mathbf{2 0 0 0}$ \\
\hline $\mathrm{A}(\phi)$ & 2.27 & 2.35 & 1.82 & 1.42 & 1.68 \\
$\mathrm{~B}\left(\sigma^{2}\right)$ & 0.68 & 0.62 & 0.60 & 0.60 & 0.64 \\
$\mathrm{C}\left(\phi_{i}\right)$ & 2.26 & 2.34 & 1.80 & 1.39 & 1.71 \\
$\mathrm{D}\left(\phi\left(p_{i}\right)\right)$ & 2.28 & 2.29 & 1.82 & 1.42 & 1.73 \\
$\mathrm{E}\left(\sigma_{e, i}^{2}\right)$ & 0.72 & 0.64 & 0.62 & 0.60 & 0.72 \\
\hline
\end{tabular}

${ }^{\text {a }} D$ : Minus two times (the quasi-likelihood for the specified generalized linear mixed model minus the quasi-likelihood for the saturated model), conditional on the random effects in the specified model. $v$ : Degrees of freedom, i.e., number of observations minus the number of fixed-effects parameters.

${ }^{\mathrm{b}}$ Models A, C, and D correspond to equation 1 with overdispersion represented by a parameter $\phi$. This term was either constant for all treatments (Model A $[\phi]$ ), dependent on treatment $\left(\mathrm{C}\left[\phi_{i}\right]\right)$, or a binary power-law function of incidence $\left(\mathrm{D}\left[\phi\left(p_{i}\right)\right]\right.$; see eq. 4). Models $\mathrm{B}$ and $\mathrm{E}$ correspond to equation 2 with variability within plots represented by a variance term $\sigma_{e}^{2}$. This term was either constant for all treatments $\left(\mathrm{B}\left[\sigma_{e}^{2}\right]\right)$ or dependent on treatment $\left(\mathrm{E}\left[\sigma_{e, i}^{2}\right]\right)$. 
does not have a physical interpretation when $b^{\prime}>0$.

Residual plots revealed substantial difference in the fit of the models based on equation 1 (Models A, C, D) and equation 2 (Models B and E). Plots are shown in Figure 1 for Models A, B, and D. The plots for Model $\mathrm{C}$ were very similar to those for Model A, and plots for Model E were very similar to those for Model B (L. V. Madden, unpublished). Generally, a random scatter of standardized Pearson residuals was obtained with Model A, as well as for Model D. On the other hand, there was always an undesirable trend in residuals with Model B, with increasing residuals as the estimated linear predictor $[\hat{\eta}=$ $\operatorname{logit}(\hat{p})]$ increased.

Treatment evaluations. Model choice had a large effect on the $F$ test for treatment effects in some years and not in others (Table 4). When mean disease incidence varied greatly with treatment (e.g., 1997, 2000 [Table 1]), the significance level of the $F$ test $(P)$ was small $(<0.001)$ and unaffected by the selected model. However, the largest $F$ statistic corresponded to Models $\mathrm{C}$ and D in these years. When there was no obvious difference in treatment means (1996), $P$ values were all above 0.05 , although the largest $F$ values were for Models C and D. In 1998 and 1999, $P$ values varied considerably with model choice, and conclusions regarding significance could be affected by the model being used (Table $4)$. These years were characterized by the lowest (1998) and highest (1999) overall mean disease incidence (Table 1). In most cases with a significant $F$ test for treatment, accounting for possible variable heterogeneity using Models C and D (eq. 1) resulted in higher $F$ statistics and lower $P$ values than when the corresponding fixed-heterogeneity Model A was used. Accounting for variable heterogeneity using Model E (eq. 2) did not necessarily result in a higher $F$ statistic compared with Model B.

Table 3. Estimates of the overdispersion parameter $(\phi)$ from Model A, within-plot sampling variance $\left(\sigma_{e}^{2}\right)$ from Model $\mathrm{B}$, and $A$ and $b^{\prime}$ overdispersion parameters from Model $\mathrm{D}$, for generalized linear mixed models (GLMMs) ${ }^{\mathrm{a}}$ fitted to the strawberry leaf blight disease incidence data

\begin{tabular}{lcclcc}
\hline & \multicolumn{5}{c}{ Year } \\
\cline { 2 - 6 } Term & $\mathbf{1 9 9 6}$ & $\mathbf{1 9 9 7}$ & $\mathbf{1 9 9 8}$ & $\mathbf{1 9 9 9}$ & $\mathbf{2 0 0 0}$ \\
\hline$\phi$ & 2.11 & 2.20 & 1.91 & 1.42 & 1.64 \\
$\sigma^{2}{ }_{e}$ & 0.48 & 0.77 & 0.72 & 0.61 & 0.28 \\
$A$ & 30.2 & 40.6 & 9.0 & 69.2 & 6.0 \\
$b^{\prime}$ & 0.61 & $0.61^{* \mathrm{~b}}$ & $0.29^{*}$ & $0.75^{*}$ & 0.28 \\
\hline
\end{tabular}

${ }^{a}$ Models A, C, and D correspond to equation 1 with overdispersion represented by a parameter $\phi$. This term was either constant for all treatments (Model A $[\phi]$ ), dependent on treatment $\left(C\left[\phi_{i}\right]\right)$, or a binary power-law function of incidence ( $\mathrm{D}\left[\phi\left(p_{i}\right)\right]$; see eq. 4$)$. Models $\mathrm{B}$ and $\mathrm{E}$ correspond to equation 2 with variability within plots represented by a variance term $\sigma^{2}{ }_{e}$. This term was either constant for all treatments $\left(\mathrm{B}\left[\sigma^{2}{ }_{e}\right]\right)$ or dependent on treatment $\left(\mathrm{E}\left[\sigma^{2} e_{e, i}\right]\right)$.

b Asterisk (*) indicates that estimate of $b^{\prime}$ is at least $2.64 \times$ larger than its standard error. The standard error is estimated by the MIXED procedure of SAS based on large-sample theory. At moderate sample sizes in general, and GLMMs in particular, the sampling distribution of any estimated variance term likely is skewed (40). Thus, the ratio of a variance estimated and its standard error should just be a rough guide to significance.
Model choice had some effect on the SEs of the estimated linear predictors, but the effect was only substantial for treatments with large standard errors (i.e., treatments 5 and 7) in 1997 (Table 6). For these two treatments, the standard errors based on assumed fixed residual variance (Model A) were considerably larger than for the other models. Conversely, for treatments with relatively low standard errors (e.g., treatment 2), Model A resulted in the lowest SE (Table 6) among the five models, although the differences among models were small. In general, the SEs corresponding to the five models were very similar for many treatments, especially treatments 1 to 4 and 8 .

The percentage of significant pairwise differences of estimated linear predictors was high for years with high $F$ statistics (1997 and 2000) and low in years with small $F$ statistics (1996) (Table 7), as expected. However, the percentages were generally not related to model choice within years, even in years with substantial differences in $F$ statistics among models. Only in 1999 were the percentages among models noticeably related to $F$ statistics, with Model E exhibiting the lowest $F$ (highest $P$ ) and lowest percentage of significant differences (Table 7).

Treatment-specific variation in 1997. Model $\mathrm{C}$ resulted in estimates of the treatment-specific overdispersion terms $\left(\hat{\phi}_{i}\right)$ ranging from 0.7 to 3.5 in 1997 (Table 6). This compares with $\hat{\phi}=2.2$ based on Model A (Table 2). One can also estimate treatment-specific $\phi$ values using the estimates of $A$ and $b^{\prime}$ from Model D ( $\hat{\phi}\left(\hat{p}_{i}\right)$; see eq. 4 and Table 2). These $\hat{\phi}$ s ranged from 0.95 to 3.3 . By definition, $\hat{\phi}\left(\hat{p}_{i}\right)$ increases with $\hat{p}$ up to 0.5 (or up to a logit of 0 ) and then declines (26); in contrast, there is no constraint on how $\hat{\phi}_{i}$ changes with $\hat{p}_{i}$ (or logits), although the $\hat{\phi}_{i} \mathrm{~s}$ showed the same general trend with the estimated linear predictors as did the $\hat{\phi}\left(\hat{p}_{i}\right)$ s (Table 6). The rank order of the estimates of $\phi_{i}$, however, was not the same, with the largest and smallest dispersion values for these two models corresponding to different treatments. Across all 5 years, however, there was a general straight-line relationship between the two estimates of treatment-specific $\phi$, but there was variation in the relationship (Fig. 2).

Assessment of the $\hat{\phi}_{i}$ s can explain differences in calculated SEs. Because the estimates of $\phi$ for treatments 5 and 7 based on either Model C or D were considerably below the single $\hat{\phi}$ calculated with Model A, the SEs calculated with Model A were too large (assuming that Model C or D is correct), because the SE is dependent on $\phi$. Likewise, because $\hat{\phi}$ for treatment $2\left(\hat{\phi}_{2}\right)$ based on either Model C or D (3.5 and 2.9, respectively) was considerably above the single $\hat{\phi}$ of Model A, the SE calculated with Model A was too small. Since several treatments 
(i.e., 1, 3, 4, and 8) had similar $\hat{\phi}_{i}$ s, their SEs also were very similar.

Estimates of the treatment-specific within-plot variance for Model $\mathrm{E}\left(\hat{\sigma}_{e, i}^{2}\right)$ ranged from 0.0 to 1.3 in 1997 (Table 6). These can be compared with the single variance estimate of 0.77 determined with Model B (Table 3). Although the treat- ments with the largest and smallest $\hat{\sigma}_{e, i}^{2}$ values (2 and 5) also had the largest and smallest $\hat{\phi}_{i}$ s (based on Model C), the agreement did not always hold for other
Model A
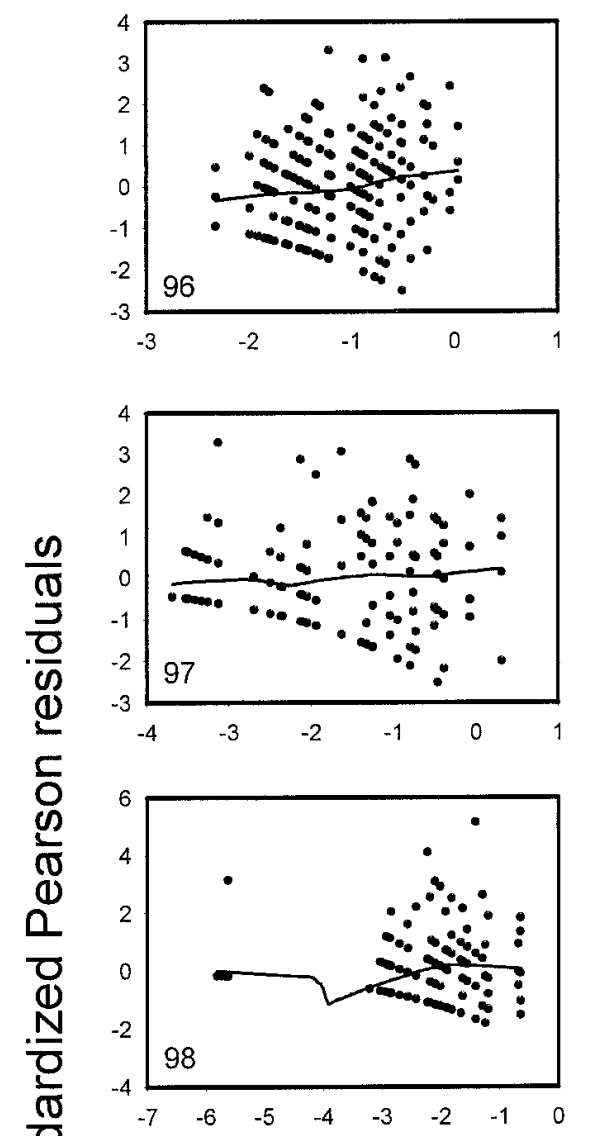

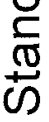
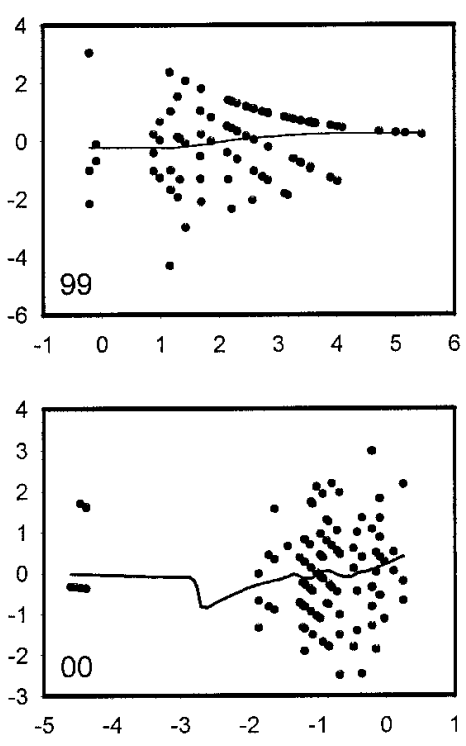

Model B
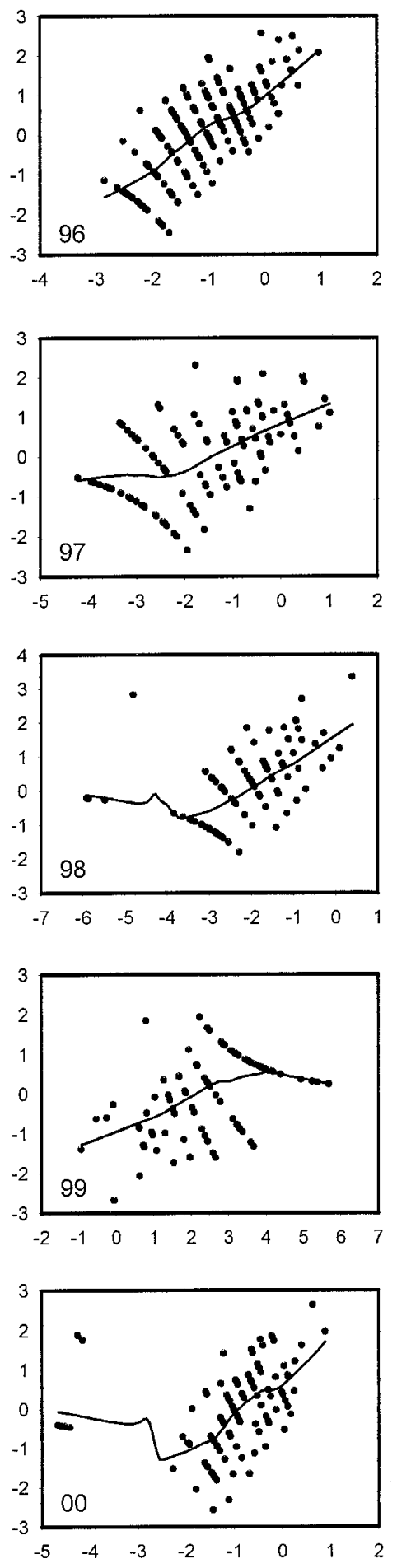

Model D
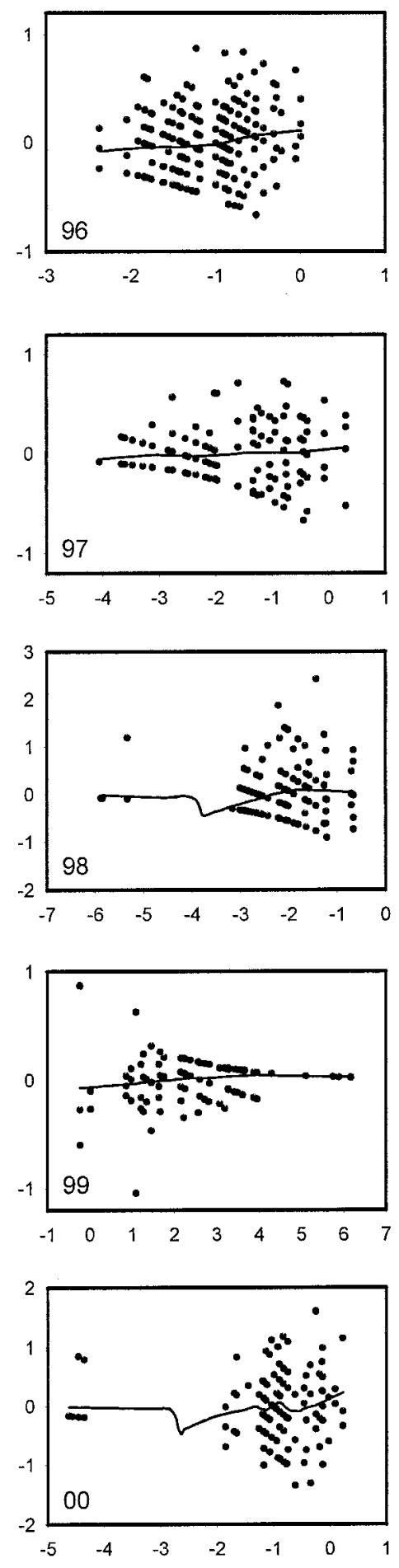

Estimated linear predictors (logits)

Fig. 1. Standardized Pearson residuals $(r)$ versus estimated linear predictors (i.e., least squares means or logits of estimated probability of a diseased leaflet) based on three generalized linear mixed models (GLMMs) fitted to data sets of Phomopsis leaf blight disease incidence over 5 years. Model A corresponds to equation 1 , with a single overdispersion parameter $(\phi)$. Model B corresponds to equation 2, with a single within-plot sampling variance $\left(\sigma_{e}^{2}\right)$. Model D corresponds to equation 1, but with the overdispersion parameter equal to a function of disease incidence $\left[\phi\left(p_{i}\right)\right.$; eq. 4]. The scale difference in residuals between Models A and D is not relevant because the $A$ parameter in equation 4 has no physical meaning when $b^{\prime}>0$. Curves represent a locally weighted scatterplot smoother (LOWESS) function fitted to the residuals. 
treatments. For instance, $\hat{\phi}_{7}$ was the second smallest overdispersion parameter with Model C, but $\hat{\sigma}_{e, 7}^{2}$ was the second largest within-plot sampling variance with Model E.

\section{DISCUSSION}

In this study, we have successfully fitted GLMMs, with either fixed or treatmentdependent variability, to five data sets involving the incidence of strawberry leaflets with Phomopsis leaf blight symptoms. The iterative REPL model-fitting procedure (42) converged in all cases, and it was possible to estimate the fixed- (i.e., treat- ment) and random- (i.e., block, experimental variability) effect terms for all experiments. When fitting Model A (eq. 1) to the data, the magnitude of the deviance conditional on the random effects $(D)$ indicated that there was overdispersion (greater variability than expected for a binomial distribution). Applying generalized linear models to binary data, one typically first assumes that the data have a binomial distribution and then modifies or expands the model if there is evidence for overdispersion. There are three general approaches for this expansion with generalized linear models (with variations of each) $(5,19)$,

Table 4. $F$ statistic and significance level $(P)$ for the test of the effect of treatment on the incidence of Phomopsis leaf blight of strawberry over 5 years based on five generalized linear mixed models (GLMMs) fitted to the data ${ }^{a}$

\begin{tabular}{lccccc}
\hline & \multicolumn{5}{c}{ Year } \\
\cline { 2 - 6 } Model & $\mathbf{1 9 9 6}$ & $\mathbf{1 9 9 7}$ & $\mathbf{1 9 9 8}$ & $\mathbf{1 9 9 9}$ & $\mathbf{2 0 0 0}$ \\
\hline A & $1.82(0.090)^{\mathrm{b}}$ & $8.69(<0.001)$ & $2.26(0.059)$ & $2.38(0.040)$ & $5.75(0.001)$ \\
B & $1.79(0.097)$ & $8.81(<0.001)$ & $2.60(0.033)$ & $2.38(0.040)$ & $6.75(<0.001)$ \\
C & $1.87(0.081)$ & $10.7(<0.001)$ & $3.38(0.010)$ & $3.44(0.006)$ & $8.32(<0.001)$ \\
D & $1.96(0.067)$ & $9.74(<0.001)$ & $3.17(0.014)$ & $3.40(0.007)$ & $7.80(<0.001)$ \\
E & $1.72(0.111)$ & $9.37(<0.001)$ & $2.87(0.022)$ & $2.07(0.070)$ & $7.64(<0.001)$ \\
\hline
\end{tabular}

${ }^{a}$ Models A, C, and D correspond to equation 1 with overdispersion represented by a parameter $\phi$. This term was either constant for all treatments (Model A $[\phi]$ ), dependent on treatment $\left(C\left[\phi_{i}\right]\right.$ ), or a binary power-law function of incidence ( $\mathrm{D}\left[\phi\left(p_{i}\right)\right]$; see eq. 4). Models B and $\mathrm{E}$ correspond to equation 2 with variability within plots represented by a variance term $\sigma_{e}^{2}$. This term was either constant for all treatments $\left(\mathrm{B}\left[\sigma_{e}^{2}\right]\right)$ or dependent on treatment $\left(\mathrm{E}\left[\sigma_{e, i}^{2}\right]\right)$.

${ }^{\mathrm{b}} F(P)$.

Table 5. Estimated experimental error variance (notationally equal to the interaction of treatment and block; $\hat{\sigma}_{\tau B}^{2}$ ) for the fit of generalized linear mixed models (GLMMs) to the strawberry leaf blight data over 5 years ${ }^{\mathrm{a}}$

\begin{tabular}{llllll}
\hline & \multicolumn{5}{c}{ Year } \\
\cline { 2 - 6 } Model & $\mathbf{1 9 9 6}$ & $\mathbf{1 9 9 7}$ & $\mathbf{1 9 9 8}$ & $\mathbf{1 9 9 9}$ & $\mathbf{2 0 0 0}$ \\
\hline A & 0.22 & 0.22 & 0.47 & 0.92 & 0.06 \\
B & 0.25 & 0.28 & 0.56 & 0.89 & 0.04 \\
C & 0.24 & 0.25 & 0.56 & 1.18 & 0.05 \\
D & 0.21 & 0.31 & 0.49 & 0.94 & 0.04 \\
E & 0.27 & 0.26 & 0.57 & 0.91 & 0.05 \\
\hline
\end{tabular}

${ }^{\mathrm{a}}$ Models A, C, and D correspond to equation 1 with overdispersion represented by a parameter $\phi$. This term was either constant for all treatments (Model A $[\phi]$ ), dependent on treatment $\left(C\left[\phi_{i}\right]\right)$, or a binary power-law function of incidence ( $\mathrm{D}\left[\phi\left(p_{i}\right)\right]$; see eq. 4). Models B and E correspond to equation 2 with variability within plots represented by a variance term $\sigma^{2}{ }_{e}$. This term was either constant for all treatments $\left(\mathrm{B}\left[\sigma_{e}^{2}\right]\right)$ or dependent on treatment $\left(\mathrm{E}\left[\sigma_{e, i}^{2}\right]\right)$.

and forms of two of these can be performed with GLMMs. The first is to assume that the residual variance (or variation in $p$ ) is inflated relative to the binomial by a factor $\phi$, estimate the term, and use $\hat{\phi}$ to adjust all test statistics. This is the approach taken with Model A for GLMMs. The second is to use a model with a random-effects term, with an assumed normal distribution, for the lowest level in a hierarchy (16). This is the approach taken with Model B to account for variability of the logit of $p$ within experimental units (plots). Such an approach follows naturally for overdispersion in mixed models, because other random terms (e.g., block, experimental error) are already represented as being normally distributed and additive on a logit scale. The third approach also deals with a random effect, but uses a nonnormal continuous distribution to represent the variation in the probability of disease among the samples within the experimental units, not the variation in the logits (3). One specific formulation of this with generalized linear models results in the beta-binomial distribution (13) to describe disease incidence (14). However, there is no direct way of fitting a beta-binomial-type model using the REPL method implemented in \%GLIMMIX. Recent advances in hierarchical generalized linear models (18) and nonlinear mixed models (34) allow for some expansions of this form for certain (uncomplicated) experimental designs, but are not yet as easily implemented for general usage.

Selecting between GLMMs with either an overdispersion parameter (e.g., Model A) or a random-effect variance term for within-plot variation (e.g., Model B) is not clear cut under many circumstances $(10,19,20,33)$. With the five strawberry leaf blight data sets, there is some (weak) evidence to favor Model A over B. First, the conditional deviance divided by the degrees of freedom, $D / v$, was considerably smaller than 1 (the assumed $\phi$ ) for Model $\mathrm{B}$, indicating that adding the $e_{i j k}$ term may

Table 6. Least squares means (estimated linear predictors [logits]) and standard errors of the least squares means for the eight treatments in 1997, and, where relevant, the treatment-specific estimated parameters for variability, for five generalized linear mixed models fitted to strawberry leaf blight data ${ }^{a}$

\begin{tabular}{|c|c|c|c|c|c|c|c|c|c|c|c|c|c|}
\hline \multirow[b]{3}{*}{ Treatment } & \multicolumn{13}{|c|}{ Modelb } \\
\hline & \multicolumn{2}{|c|}{$\mathbf{A}$} & \multicolumn{2}{|c|}{ B } & \multicolumn{3}{|c|}{ C } & \multicolumn{3}{|c|}{ D } & \multicolumn{3}{|c|}{$\mathbf{E}$} \\
\hline & Mean & SE & Mean & SE & Mean & SE & $\phi$ & Mean & SE & $\phi(p)$ & Mean & SE & $\sigma_{e, i}^{2}$ \\
\hline 1 & -0.88 & 0.302 & -0.98 & 0.357 & -0.88 & 0.332 & 3.00 & -0.88 & 0.355 & 2.97 & -0.99 & 0.353 & 0.82 \\
\hline 2 & -0.96 & 0.303 & -1.09 & 0.358 & -0.95 & 0.347 & 3.51 & -0.96 & 0.355 & 2.91 & -1.14 & 0.388 & 1.27 \\
\hline 3 & -1.79 & 0.340 & -1.96 & 0.377 & -1.80 & 0.350 & 2.21 & -1.80 & 0.371 & 2.14 & -1.96 & 0.371 & 0.79 \\
\hline 4 & -1.79 & 0.340 & -2.02 & 0.382 & -1.80 & 0.354 & 2.29 & -1.81 & 0.374 & 2.14 & -2.05 & 0.390 & 0.97 \\
\hline 5 & -3.37 & 0.532 & -3.44 & 0.466 & -3.39 & 0.369 & 0.71 & -3.39 & 0.421 & 0.95 & -3.38 & 0.411 & 0.00 \\
\hline 6 & -2.42 & 0.390 & -2.55 & 0.400 & -2.43 & 0.356 & 1.45 & -2.44 & 0.388 & 1.58 & -2.50 & 0.364 & 0.40 \\
\hline 7 & -3.50 & 0.557 & -3.74 & 0.504 & -3.54 & 0.476 & 1.38 & -3.62 & 0.440 & 0.82 & -3.80 & 0.520 & 1.05 \\
\hline 8 & -0.15 & 0.292 & -0.18 & 0.351 & -0.15 & 0.317 & 2.86 & -0.15 & 0.350 & 3.33 & -0.17 & 0.335 & 0.66 \\
\hline
\end{tabular}

${ }^{a}$ Logits of the actual proportion of diseased leaflets (see Table 1) for treatment numbers 1 to 8 are: -0.85 (\#1), -0.94 (\#2), -1.73 (\#3), -1.73 (\#4), -3.48 (\#5), - 2.44 (\#6) -3.48 (\#7), and $-0.16(\# 8)$.

${ }^{\mathrm{b}}$ Models A, C, and D correspond to equation 1 with overdispersion represented by a parameter $\phi$. This term was either constant for all treatments (Model A $[\phi])$, dependent on treatment $\left(\mathrm{C}\left[\phi_{i}\right]\right)$, or a binary power-law function of incidence $\left(\mathrm{D}\left[\phi\left(p_{i}\right)\right]\right.$; see eq. 4$)$. Models B and $\mathrm{E}$ correspond to equation 2 with variability within plots represented by a variance term $\sigma_{e}^{2}$. This term was either constant for all treatments $\left(\mathrm{B}\left[\sigma^{2}{ }_{e}\right]\right)$ or dependent on treatment $\left(\mathrm{E}\left[\sigma^{2}{ }_{e, i}\right]\right)$. 
have "overcorrected" for the variability within plots. However, the statistical properties of $D$ are not fully known for GLMMs (5), so it is not certain exactly how small $D$ 's should be to fully reflect underdispersion. Second, use of Model B resulted in a bias in the estimates of the probability of disease for the individual treatments, but the bias was slight. Third, the plots of standardized Pearson residuals $(r)$ versus the estimated linear predictors $(\hat{\eta})$ exhibited strong patterns with Model $\mathrm{B}$, but not with Model $\mathrm{A}$. This may reflect the bias in the estimate of $\eta$ (23). Other types of residuals - such as "unstandardized" Pearson residuals, deviance residuals, and standardized deviance residuals (5)-showed the same patterns (L. V. Madden, unpublished). Liang and McCullagh (20) and Lee and Nelder (19) give fuller accounts of using some specialized residual plots to discriminate between models.

Given that within-plot or within-field variability can be modeled as a function of disease incidence through the binary power law (eq. 3) $(12,15,26,35,39,43)$, it is natural to ask whether the variability corresponding to a GLMM fitted to incidence data depends on levels of the fixed factors (e.g., treatment). Results for Models $\mathrm{C}$ and E showed that residual variation $\left(\hat{\phi}_{i}\right)$ and within-plot sampling variance $\left(\hat{\sigma}_{e, i}^{2}\right)$, respectively, depended on treatment (Table 6; Madden, unpublished). As demonstrated for 1997, the largest $\hat{\phi}_{i}$ values often were associated with means $(\hat{p})$ near 0.5 (or logits near 0 ), and declined as incidence approached 0 (or logits became more negative). However, the overall evidence of a better fit by these two models compared with the simpler Models A and B was equivocal. Values of $D / v$ for Model C were very similar to values for Model $\mathrm{A}$, and values of $D / v$ for Model $E$ were very similar to values for Model B. Also, Model E was the most difficult one to fit because one has to provide initial estimates of the treatment-specific variances prior to estimating the terms (33). Although convergence was always achieved with our data even when initial estimates were far from the "true" values, experience shows with other data sets that more than one set of initial estimates may have to be tried in order to achieve convergence with the REPL procedure (L. V. Madden, unpublished). Initial estimates of the $\phi_{i}$ s are not required when fitting Model $\mathrm{C}$ to data, making the model fitting slightly less cumbersome than fitting Model E.

An efficient method of representing variability as a function of mean incidence is to directly incorporate the binary power law into the analysis $(14,25)$. This was done here in the context of GLMMs when fitting Model D to the data (see eq. 4). Only one additional term $\left(b^{\prime}\right)$ is required to account for the treatment-dependent variability instead of the additional $t-1$ terms for Model C. For instance, with the treatments in 1997 (Table 6), eight variance parameters were required to account for treatment-dependent variability using Model C $\left(\phi_{i}, i=1, \ldots, 8\right)$, which is seven more parameters than the single $\phi$ for Model A; with Model D, two variance parameters are required $\left(A\right.$ and $\left.b^{\prime}\right)$ instead of the single term for Model A. Because the degree of overdispersion in Model D, $\phi(p)$, depends on both estimated probability of disease $(\hat{p})$ and the exponent $\hat{b}^{\prime}$, Model D is somewhat different from the others considered, but the difference has no direct relevance in the model evaluations. Model D did result in acceptable residual plots (as did Model A) for the strawberry leaf blight data, and the estimated $b^{\prime}$ was generally larger than 0 . However, there was no clear improvement in the model fit compared to Model A based on the values of $D / v$. Fitting Model D is somewhat more tedious than fitting Model A or C, because a two-step procedure is necessary, but we found no difficulty in obtaining parameter estimates for the five data sets with the REPL algorithm. Estimates of $b\left(=\hat{b}^{\prime}+1\right)$ obtained in this study (Table 2 ) were comparable to, but somewhat greater than, estimates obtained in other studies involving the same disease. For instance, in an analysis of heterogeneity of Phomopsis leaf blight in commercial strawberry fields in 1996 and 1997 (but not including the plots analyzed here), Turechek and Madden (39) estimated $b$ as 1.2. That study

Table 7. Percentage of pairwise differences of estimated linear predictors (i.e., least squares means or logits of estimated probability of disease for each treatment) that were significantly different from 0 based on $t$ tests of linear contrasts ${ }^{\mathrm{a}}$ for strawberry leaf blight data over 5 years ${ }^{\mathrm{b}}$

\begin{tabular}{lccccc}
\hline & \multicolumn{5}{c}{ Year } \\
\cline { 2 - 6 } Model & $\mathbf{1 9 9 6}$ & $\mathbf{1 9 9 7}$ & $\mathbf{1 9 9 8}$ & $\mathbf{1 9 9 9}$ & $\mathbf{2 0 0 0}$ \\
\hline A & 22 & 61 & 39 & 29 & 53 \\
B & 20 & 57 & 36 & 24 & 56 \\
C & 21 & 54 & 33 & 31 & 56 \\
D & 24 & 54 & 39 & 33 & 58 \\
E & 16 & 61 & 39 & 20 & 51 \\
\hline
\end{tabular}

${ }^{a} t$ test is defined as the difference of the estimated linear predictors for a pair of treatments divided by the standard error of the difference. Standard error of the difference (SED) was calculated based on a linear combination of the variance components (23).

${ }^{\mathrm{b}}$ Models A, C, and D correspond to equation 1 with overdispersion represented by a parameter $\phi$. This term was either constant for all treatments (Model A $[\phi])$, dependent on treatment $\left(C\left[\phi_{i}\right]\right)$, or a binary power-law function of incidence ( $\mathrm{D}\left[\phi\left(p_{i}\right)\right]$; see eq. 4). Models $\mathrm{B}$ and $\mathrm{E}$ correspond to equa-

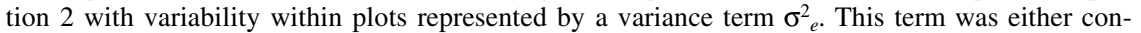
stant for all treatments $\left(\mathrm{B}\left[\sigma_{e}^{2}\right]\right)$ or dependent on treatment $\left(\mathrm{E}\left[\sigma_{e, i}^{2}\right]\right)$.

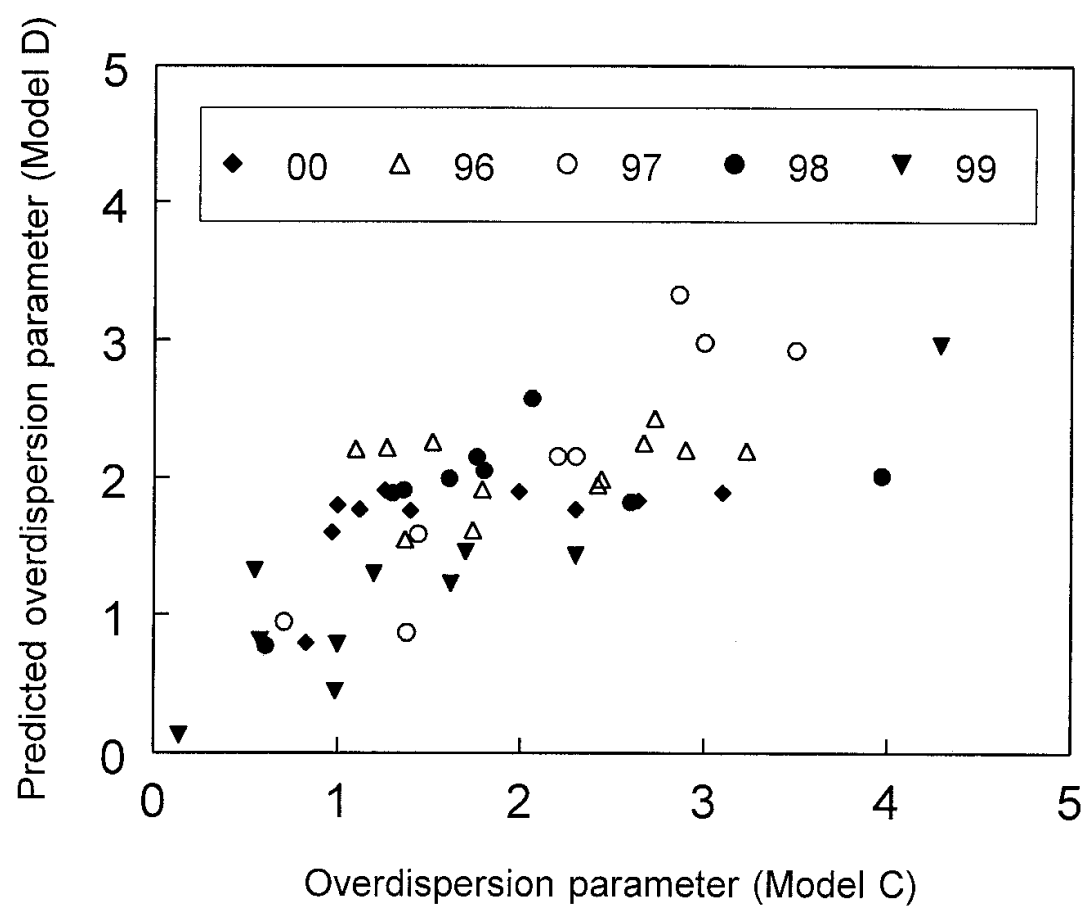

Fig. 2. Relationship between estimates of the treatment-dependent overdispersion parameter for Model C $\left(\hat{\phi}_{i}\right)$ and predicted overdispersion parameter for Model D based on the estimate of the probability of disease for the $i$ th treatment $\left[\phi\left(\hat{p}_{i}\right)\right.$; eq. 4]. Results are for generalized linear mixed models (GLMMs) fitted to strawberry leaf blight data sets over each of 5 years. Data points are labeled by year. 
involved determining $x$ and $\operatorname{Var}(x)$ based on 75 sampling units in each field, and fitting the logarithmic version of equation 3 to the data using linear regression analysis. In the analysis reported here, which involved the residuals of a GLMM fitted to data from a planned experiment, estimates of $b$ were about 1.6 (i.e., $0.6+1$; Table 2). Analysis of different data sets and the number of sampling units per plot (field) could be the causes of the difference. Plus, it is known that the model-fitting method affects the estimate of $b$ for the power law (27). Interestingly, when we fitted a version of Model D (with $\phi$ dependent on the incidence of the plot) to the grape downy mildew data described in Hughes and Madden (14), we obtained an estimate of $b$ of $1.4\left(\hat{b}^{\prime}=0.4\right)$ (L. V. Madden, unpublished). This is the same as the estimate of $b$ obtained by Madden et al. (27) directly from the variances for each plot (independent of the experimental design).

The purpose of many field experiments is to estimate the fixed effects, such as fungicide treatment in this study. In these cases, estimation of the random effects is not an objective per se, rather, it is done only to allow the proper estimation and testing of fixed effects (23). Even though there were differences in the fit of the five GLMMs to the Phomopsis data sets, especially in terms of some estimated variances (Tables 2 and 5), the choice of model only had an influence on tests of fungicide treatment in some years, and seldom had an influence on the percentage of pairwise differences of estimated linear predictors that were significant. In particular, $F$ tests were unaffected by the model when differences in incidence were large, such that the significance level $(P)$ was always less than 0.001 . However, in years when the differences in incidence between many of the treatments were smaller, model choice did affect $P$.

In conclusion, models based on overdispersion $(\phi>1)$ and within-plot sampling variation $\left(\sigma_{e}^{2}>0\right)$ were both reasonable for analyzing Phomopsis leaf blight data sets over 5 years. There was some evidence favoring models based on equation 1 (Models A, C, and D) over the models based on equation 2 (Models B and E), such as residual plots, but the effects on tests for treatment often were minor. Although there was evidence for heterogeneity being dependent on treatment or incidence, as expected, the improvement in the model fit was not necessarily large, nor did it necessarily change conclusions regarding treatment effects. Apparently, the effects of nonfixed heterogeneity were not great enough to influence many fixed-effects results. Because of its relative ease of use, we recommend Model A as the first choice in analyzing the effects of fixed factors on disease incidence. Then, if $F$ tests are marginal (e.g., $P=0.03$ to 0.10 ), one should consider expanding the model for variable heterogeneity, because it is under these circumstances that model choice can have an impact on analysis of fixed effects. When there are many treatments or several fixed-effect factors, it will be more efficient to use Model D, based on the binary power law, than to use Model C. This is because of the large number of variance parameters that would need to be estimated with Model C. Based on the results here, and those reported by Piepho (33), it is clear that a GLMM is a useful alternative to ANOVA for analyzing disease incidence data when there is more than one source of variability. It should be emphasized, however, that the statistical theory of GLMMs is still developing $(1,23,36)$, and future research should provide greater insight on determining goodness of fit, discrimination among models, and testing of fixed and random effects.

\section{ACKNOWLEDGMENTS}

We thank Hans-Peter Piepho and Gareth Hughes for helpful comments on an earlier version of the manuscript. Salaries and research support were provided by state and federal funds appropriated to the Ohio Agricultural Research and Development Center, The Ohio State University.

\section{LITERATURE CITED}

1. Bellamy, S. L., Gibberd, R., Hancock, L., Howley, P., Kennedy, B., Klar, N., Lipsitz, S., and Ryan, L. 2000. Analysis of dichotomous outcome data for community intervention studies. Stat. Methods Med. Res. 9:135-159.

2. Breslow, N. R., and Clayton, D. G. 1993. Approximate inference in generalized linear mixed models. J. Am. Stat. Assoc. 88:8-25.

3. Candy, S. G. 2000. The application of generalized linear mixed models to multi-level sampling for insect population monitoring. Environ. Ecol. Stat. 7:217-238.

4. Carroll, R. J., and Ruppert, D. 1988. Transformation and Weighting in Regression. Chapman \& Hall, New York.

5. Collett, D. 1991. Modelling Binary Data. Chapman \& Hall, London.

6. Dunne, A., O'Hara, T., and Devane, J. 1997. Approaches to IVIVR modelling and statistical analysis. Adv. Exp. Med. Biol. 423:67-86.

7. Fung, K. Y., Lin, X., and Krewski, D. 1998. Use of generalized linear mixed models in analyzing mutant frequency data from the transgenic mouse assay. Environ. Mol. Mutagenesis 31:48-54.

8. Funt, R. C., Ellis, M. A., and Welty, C. 1997. Midwest Small Fruit Pest Management Handbook. Ohio State University Ext. Bull. 861. Columbus, $\mathrm{OH}$.

9. Gilligan, C. A. 1986. Use and misuse of the analysis of variance in plant pathology. Pages 225-261 in: Advances in Plant Pathology, Vol. 5. Academic Press, New York.

10. Guo, G., and Zhao, H. 2000. Multilevel modeling for binary data. Annu. Rev. Sociol. 26:441-462.

11. Henderson, C. R. 1984. Applications of Linear Models in Animal Breeding. University of Guelph Press, Guelph, Ontario.

12. Hughes, G., and Madden, L. V. 1992. Aggregation and incidence of disease. Plant Pathol. 41:657-660.

13. Hughes, G., and Madden, L. V. 1993. Using the beta-binomial distribution to describe aggregated patterns of disease incidence. Phytopathology 83:759-763.

14. Hughes, G., and Madden, L. V. 1995. Some methods allowing for aggregated patterns of disease incidence in the analysis of data from designed experiments. Plant Pathol. 44:927943.

15. Hughes, G., McRoberts, N., Madden, L. V., and Nelson, S. C. 1997. Validating mathematical models of plant disease progress in space and time. IMA J. Math. Appl. Med. Biol. 14:85-112.

16. Hughes, G., Munkvold, G. P., and Samita, S 1998. Application of the logistic-normalbinomial distribution to the analysis of Eutypa dieback disease incidence. Int. J. Pest Manag. 44:35-42.

17. Kachman, S. D. 2001. An introduction to generalized linear mixed models. Pages 59-73 in: Proc. Implementation Strategies for National Beef Cattle Evaluation. NCR Coordinating Committee Symposium, Athens, GA.

18. Lee, Y., and Nelder, J. A. 1996. Hierarchical generalized linear models (with discussion). J. Roy. Stat. Soc. B 58:619-678.

19. Lee, Y., and Nelder, J. A. 2000. Two ways of modelling overdispersion in non-normal data. Appl. Stat. 49:591-598.

20. Liang, K.-Y., and McCullagh, P. 1993. Case studies in binary dispersion. Biometrics 49:623-630.

21. Lin, X., and Breslow, N. E. 1996. An analysis of correlated binomial data in logistic-normal models. J. Stat. Comput. Simul. 55:133-146.

22. Lindsey, J. K. 1999. On the use of corrections for overdispersion. Appl. Stat. 48:553-561.

23. Littell, R. C., Milliken, G. A., Stroup, W. W. and Wolfinger, R. D. 1996. SAS System for Mixed Models. SAS Institute, Cary, NC.

24. Lopes, U. V., Huber, D. A., and White, T. L. 2000. Comparison of methods for prediction of genetic gain from mass selection on binary threshold traits. Silvae Genet. 49:50-56.

25. Madden, L. V., Ellis, M. A., Lalancette, N., Hughes, G., and Wilson, L. L. 2000. Evaluation of a disease warning system for downy mildew of grapes. Plant Dis. 84:549-554.

26. Madden, L. V., and Hughes, G. 1995. Plant disease incidence: Distributions, heterogeneity, and temporal analysis. Annu. Rev. Phytopathol. 33:529-564.

27. Madden, L. V., Hughes, G., and Ellis, M. A. 1995. Spatial heterogeneity of the incidence of grape downy mildew. Phytopathology 85:269275.

28. McCullagh, P., and Nelder, J. A. 1989. Generalized Linear Models, 2nd ed. Chapman \& Hall, London.

29. McCulloch, C. E. 2000. Generalized linear models. J. Am. Stat. Assoc. 95:1320-1324.

30. Nelson, L. A. 1978. Use of statistics in planning, data analysis, and interpretation of fungicide and nematicide tests. Pages 2-14 in: Methods for Evaluating Plant Fungicides, Nematicides, and Bactericides. E. I. Zehr, G. W. Bird, K. D. Fisher, K. D. Hickey, F. H. Lewis, R. F. Line, and S. F. Rickard, eds. American Phytopathological Society, St. Paul, MN.

31. Nita, M., Madden, L. V., and Ellis, M. A 2000. Evaluation of fungicides and fungicide combinations for control of leaf blight, 1999. Fungic. Nematicide Tests 55:133.

32. Nita, M., Madden, L. V., and Ellis, M. A. 2001. Evaluation of fungicides and fungicide combinations for control of leaf blight, 2000. Fungic. Nematicide Tests 2001:SMF46 (published on-line).

33. Piepho, H.-P. 1999. Analysing disease incidence data from designed experiments by generalized linear mixed models. Plant Pathol. 48:668-674.

34. Pinheiro, J. C., and Bates, D. M. 1995. Approximations to the $\log$-likelihood function in the nonlinear mixed-effects model. J. Comput. Graph. Stat. 4:12-35.

35. Savary, S., Castilla, N. P., and Willocquet, L. 2001. Analysis of the spatio-temporal struc- 
ture of rice sheath blight epidemics in a farmer's field. Plant Pathol. 50:53-68.

36. Smith, D. M., and Kenward, M. G. 2000. Extensions of multiple linear regression. Commun. Stat.-Theory Meth. 29:2033-2053.

37. Turechek, W. W., Ellis, M. A., and Madden, L. V. 1998. Evaluation of fungicides for control of strawberry leaf blight, 1997. Fungic. Nematicide Tests 53:126.

38. Turechek, W. W., Ellis, M. A., and Madden, L. V. 1999. Evaluation of fungicides for control of leaf blight on strawberry, 1998. Fungic. Nematicide Tests 54:120.

39. Turechek, W. W., and Madden, L. V. 1999. Spatial pattern analysis of strawberry leaf blight in perennial production systems. Phytopathology 89:421-433.

40. Verbeke, G., and Molenberghs, G., eds. 1997. Linear Mixed Models in Practice; A SASOriented Approach. Lecture Notes in Statistics, No. 126. Springer-Verlag, Berlin.

41. Wolfinger, R. D. 1996. Heterogenous vari- ance-covariance structures for repeated measures. J. Agric. Biol. Environ. Stat. 1:205-230.

42. Wolfinger, R., and O'Connell, M. 1993. Generalized linear mixed models: A pseudolikelihood approach. J. Stat. Comput. Simul. 48:233-243.

43. Xu, X.-M., and Ridout, M. S. 2000. Effects of quadrat size and shape, initial epidemic conditions, and spore dispersal gradient on spatial statistics of plant disease epidemics. Phytopathology 90:738-750. 